\title{
Heart Failure with Preserved Ejection Fraction and Sudden Death: How to Identify High Risk Patients?
}

Bruno Schaaf Finkler ${ }^{1}$, Tiago Luiz Luz Leiria ${ }^{1, *}$, Clóvis Fröemming Junior ${ }^{1}$, Javier Pinos Vásquez¹, Danilo Barros Zanotta ${ }^{1}$, Marcelo Lapa Kruse ${ }^{1}$, Leonardo Martins Pires ${ }^{1}$, Gustavo Glotz de Lima ${ }^{1}$

ORCID IDS

Finkler BS (iD https://orcid.org/0000-0001-6885-3710

Leiria TLL (D) https://orcid.org/0000-0002-3905-102X

Fröemming Junior C (D) https://orcid.org/0000-0002-4770-0050

VásquezJP (D) https://orcid.org/0000-0001-7237-6136

\author{
Zanotta DB (iD https://orcid.org/0000-0001-5372-0421 \\ Kruse ML (D) https://orcid.org/0000-0002-2429-4491 \\ Pires LM (DD https://orcid.org/0000-0001-8003-8081 \\ Lima GG (D) https://orcid.org/0000-0003-0097-5206
}

\begin{abstract}
Objective: Heart failure with preserved ejection fraction corresponds to half of the cardiac failure cases, having a similar prognosis to patients with reduced ejection fraction. Cardiac sudden death is responsible to about one quarter of the death on these patients. The objective of this study is to review published studies, seeking to identify the high-risk factors for sudden cardiac death in this population. Methods: This work is a literature review on the most recent articles that assess the profile of patients with cardiac sudden death and cardiac failure with preserved ejection fraction. Results: Several trials were published involving patients with diverse characteristics that can help to identify patients with a higher risk of sudden death. The publication of risk score demonstrated that would be possible to identify patients with a $>10 \%$ risk of sudden death in 5 years, what would be equivalent to the risk of reduced ejection fraction patients eligible to implantable cardioverter-defibrillator (ICD) therapy. Trials with electrophysiological study and programmed ventricular stimulation showed a good strategy to identify low risk patients for future arrhythmic events. Conclusion: Sudden death must be a target of the therapy in the patients with preserved heart failure. Efforts should be done with the objective to identify higher risk patients and search for the better risk stratification strategy, and after that, the definition of the benefit or not of an invasive therapy such as ICD.
\end{abstract}

KEYWORDS: Sudden death; Cardiac failure; Preserved ejection fraction. 
Heart failure (HF) is a clinical syndrome characterized by a set of signs and symptoms caused by structural or functional cardiac abnormalities, resulting in reduced cardiac output and/or increased intracardiac pressures ${ }^{1}$. This clinical syndrome represents a great impact in terms of public health around the world, being a disease with a high prevalence in the elderly population ${ }^{2}$ and responsible for a large number of hospitalizations, with an intra-hospital mortality rate of around $4 \%{ }^{3}$ which can reach $10 \%$ in the first month after discharge ${ }^{4}$. It is estimated that at least half of patients with $\mathrm{HF}$ are classified as $\mathrm{HF}$ with preserved ejection fraction (EF) ${ }^{5}$, having as clinical markers of the disease the presence of signs and symptoms of HF, EF preserved ( $\geq 50 \%$ or between $40-49 \%$ for mid-range $\mathrm{HF}$ ), elevation of natriuretic peptides, objective evidence of structural or functional cardiac changes and increased filling pressures in the left ventricle ${ }^{1}$.

It is estimated that the annual all-cause mortality is similar among patients with $\mathrm{HF}$ with preserved (HFpEF) and reduced ejection fraction (HFrEF), around 25-30\% per year ${ }^{6,7}$, but a meta-analysis with 42,000 patients showed a lower trend of death among patients with $\mathrm{HFpEF}^{8}$. Among the causes of death, the largest studies involving patients with HFpEF estimate that sudden cardiac death (SCD) is responsible for about $40 \%$ of cardiovascular deaths, and can reach up to $20 \%$ of all causes of death ${ }^{9-11}$. The risk stratification and indication of implantable cardioverter-defibrillator (ICD) in patients with HFrEF is well-established by guidelines, but there is still no consensus regarding its indication and investigation of measures for prevention of SCD among patients with HFpEF.

One of the first large trials involving patients with HFpEF was the Candesartan in Heart Failure Assessment of Reduction in Mortality and Morbidity (CHARM) study ${ }^{10}$, in which a total of 134 events attributed as SCD in 37 months of follow-up were demonstrated, representing $28 \%$ of all deaths and $39 \%$ of cardiovascular deaths. Subsequently, two large studies involving drugs versus placebo in patients with HFpEF demonstrated a similar incidence of SCD: the Irbesartan in Heart Failure with Preserved Ejection Fraction Study (I-PRESERVE) with 231 SCD events, characterizing $26 \%$ of all deaths and $43 \%$ of cardiovascular death ${ }^{1}$ and the Treatment of Preserved Cardiac Function Heart Failure with an Aldosterone Antagonist (TOPCAT) with 128 SCD events, totaling 24\% of all deaths and $38 \%$ of cardiovascular deaths in a 40 -month follow-up 9.

In the community-based cohorts published in 2012, the incidence of SCD was lower in patients with HFpEF compared to HFrEF. The Minnesota heart survey cohort of 2,203 patients presented an $11 \%$ incidence of sudden death in 5 years of evaluation. In a multivariate analysis of the characteristics of patients with SCD, age, male gender, chronic kidney disease and length of hospitalization were independent predictors of SCD among patients with $\mathrm{HFpEF}^{12}$. The Japanese JCARE-CARD registry cohort with 2,675 patients showed an incidence of SCD of $10,7 \%{ }^{13}$.

In a subanalysis of the TOPCAT study, with only patients from the centers of the American continents, assessing the profile of patients who presented the event of SCD or aborted cardiorespiratory arrest, it was shown that these patients tend to be younger ( $70 \pm 9$ vs $75 \pm 9$ years), mostly men, with higher body mass index (BMI) and higher rates of diabetes mellitus. In this study, the ejection fraction did not differ significantly among patients who had SCD or aborted cardiorespiratory arrest, as well as the incidence of previous infarction or coronary artery disease. In the final regression model of competing risks, the variables male gender, diabetes mellitus in insulin use, smoking and glomerular filtration rate $(\mathrm{GFR})<60 \mathrm{ml} / \mathrm{min} / 1,73 \mathrm{~m}^{2}$ were independent predictors of SCD. The value of B-type natriuretic peptide (BNP) and NT-proBNP alone was not a predictor of $\mathrm{SCD}^{14}$.

The analysis of causes of death was also performed in a subanalysis of the I-PRESERVE study. They demonstrated a $25 \%$ all-cause mortality over 5 years of follow-up in randomized patients for the study, with an annual incidence of $5.2 \%$. Of these deaths, $60 \%$ were from cardiovascular causes, $30 \%$ from noncardiovascular causes and $10 \%$ from unknown causes. Among the cardiovascular causes, SCD was the main cause, followed by heart failure. The presence of the variables of left branch block, lower ejection fraction, ischemic heart failure, anemia, GFR $\leq 60 \mathrm{ml} / \mathrm{min} / 1.73 \mathrm{~m}^{2}$, NT-proBNP elevation were statistically significant for association with death of cardiovascular cause ${ }^{11}$.

From the subanalysis of patients who suffered sudden death in the I-PRESERVE study, another study with a risk prediction model for patients with $\mathrm{HFpEF}$ was conducted ${ }^{15}$. A multivariate Cox regression model was generated 
to estimate regression coefficients for the selected variables. From this model, individual risk scores were calculated as the sum of the products of the individual predictive values and the estimated regression coefficients. The scores were then used to predict the individual probability of SCD over 5 years. The sample was divided into low risk $(<10 \%)$ and high risk (>10\%). Patients at risk of SCD > 10\% would have a similar risk to patients with $\mathrm{HFrEF}$ eligible for ICD therapy, according to current evidence ${ }^{16-18}$. The variable selection strategies identified age, gender (male), history of diabetes mellitus, history of acute myocardial infarction, presence of left branch block on the electrocardiogram, and lnNT-pro BNP as independent factors for SCD.

This score allowed the identification of 837 patients (24\%) classified as high risk of SCD ( $>10 \%$ in 5 years) and $2723(76 \%)$ classified as low risk of SCD ( $<10 \%$ in 5 years). The cumulative incidence of SCD in 5 years was 11 and $4 \%$ in the high- and low-risk groups, respectively. In the high-risk group, SCD was responsible for $32 \%$ of deaths, compared to $26 \%$ of the entire cohort of the I-PRESERVE study. Making a direct correlation with primary prevention studies of SCD in patients with HFrEF, the SCD-Heft study ${ }^{17}$ presented a risk of SCD of $11 \%$ in a 4.8 -year follow-up in the placebo group, demonstrating a similar risk to patients with HFpEF in the high-risk group. It is important to emphasize that the high-risk patients in this study presented, in addition to a higher risk for $\mathrm{SCD}$, a higher risk for death from other causes, including noncardiovascular causes.

This score was later applied to a validation cohort among the TOPCAT study participants ${ }^{19}$. Due to the fact that natriuretic peptide is a secondary inclusion criterion in this study, 615 patients with NT-ProBNP measurements were included for analysis of the SCD risk model and cohort validation. Based on the risk prediction model, 35\% of the patients in this cohort were classified as high risk for SCD. In a 2.9-year follow-up there were 86 deaths, of which $23(26.7 \%)$ were due to SCD and 63 (73.3\%) due to other causes. The mortality rate for SCD was 13 per 1,000 person/year. Based on the risk prediction model, 216 patients (35.1\%) were classified as high risk of SCD. These patients had a cumulative incidence of SCD in 5 years of $15.2 \%$, against $2.8 \%$ of patients in the low-risk group. The proportion of SCD for other deaths was $41 \%$ in the high-risk group vs $31 \%$ in the low-risk group. The risk of SCD was 4-fold higher in high-risk patients compared to low-risk HR 4.0 (95\% CI: 1.7, 9.6; 24 p = 0.001). Similar to the I-PRESERVE study, high-risk patients also had higher mortality from other causes.

Considering that SCD is an important cause of death among patients with HFpEF, more studies should be conducted in order to identify patients at higher risk of this event, as well as the evaluation of measures that may intervene in this outcome. Implantable cardioverter-defibrillator therapy for HFrEF patients is well established through guidelines ${ }^{1}$ and is an important part along with optimized clinical therapy. However, among $\mathrm{HFpEF}$ patients, there are not randomized clinical trials evaluating the effectiveness of this device. In a secondary analysis of Sudden Cardiac Death in Heart Failure (SCD-HEFT) patients, it was evaluated whether among patients who achieved an improvement in the ejection fraction (> 35\%) over time, ICD therapy would still show significant clinical benefit. In a 30-month follow-up it was demonstrated that even in patients with a recovered ejection fraction, the reduction in mortality was similar to those who followed with an ejection fraction $<35 \%$. The mean ejection fraction in the recovered $\mathrm{EF}$ group was $45 \%{ }^{18}$. A large population-based series demonstrated that 65 to $75 \%$ of patients who presented with cardiac arrest and died suddenly had EF $>35 \%$ in a previous examination and were not eligible for ICD implantation before the event, by the current recommendations ${ }^{20}$. This data demonstrates that the isolated evaluation of EF fails to predict a large number of high-risk patients for this outcome.

Among the risk factors evaluated noninvasively, the occurrence of nonsustained ventricular tachycardia (NSV) can be suggested as a possible factor to be researched and an aid in the identification of patients at high risk for SCD. While some studies point out that the occurrence of NSVT does not increase the risk of SCD or mortality ${ }^{21,22}$, some other studies show that its presence is related to a higher occurrence of ventricular tachycardia and/or sustained ventricular fibrillation and an independent predictor for $\mathrm{SCD}^{23,24}$. In a study published in 2019 by Zhou et al. ${ }^{25}$, it was demonstrated that the occurrence of nonsustained ventricular tachycardia with rapid frequency was an independent predictor for appropriate ICD therapy, appropriate shock, and all-cause mortality. To evaluate the occurrence of arrhythmias in patients with HFpEF, Gutierrez el al. ${ }^{26}$ used a long-stay device (14 days) to 
electrocardiographically monitor these patients. In a total of 40 patients monitored, with an average EF of 59.4\% and $44 \%$ with a history of previous ischemic heart disease, it was identified that $32 \%$ of patients had episodes of nonsustained ventricular tachycardia. All patients presented premature ventricular complexes, and of these, $7.5 \%$ had a load greater than 5\%. Patients with NSVT had lower EF, higher levels of NT-proBNP and higher degree of obesity.

The risk of arrhythmia in patients after acute myocardial infarction and preserved EF was evaluated by Gatzoulis et al. ${ }^{27}$, in a 2018 study with 575 patients. Eligible patients should have had $\mathrm{EF} \geq 40 \%$ and have more than 40 days after the acute event without evidence of active ischemia. In addition, they should have at least one noninvasive risk factor (premature ventricular complexes, nonsustained ventricular tachycardia, late potentials, corrected QT interval prolongation, increased T-wave alternans, reduced heart variability, and abnormal deceleration capacity with abnormal turbulence). Patients who met the risk criteria were referred for an electrophysiological study to perform a programmed ventricular stimulation (PVS) protocol, and if monomorphic sustained ventricular tachycardia, ventricular flutter or polymorphic ventricular tachycardia were induced, ICD therapy was offered. In a 32-month follow-up, there were no major arrhythmic events in patients who did not have any noninvasive risk factors and also in those undergoing electrophysiological study in which sustained ventricular arrhythmias were not induced. On the other hand, in the patients submitted to electrophysiological study who presented induction of sustained ventricular arrhythmias (27\% of those submitted to PVS), 9 major arrhythmic events occurred. This number corresponded to $22 \%$ of the patients of whom sustained ventricular arrhythmia was induced. This combined twostage strategy, with identification of noninvasive risk factors followed by electrophysiological study with PVS, in patients after acute myocardial infarction with $\mathrm{EF} \geq 40 \%$ demonstrated a sensitivity of $100 \%$, specificity of $93.8 \%$, a positive predictive value (PPV) of $22 \%$ and a negative predictive value (NPV) of $100 \%$.

The use of electrophysiological study and PVS was evaluated by Hilfiker et al. ${ }^{28}$ in 2015, including patients with structural or functional heart disease and/or electrical conduction abnormalities and/or after syncope or cardiorespiratory arrest, to perform PVS. The main outcome of the study was appropriate ICD activation. Sustained ventricular arrhythmia was induced in 125 patients (47.2\%) and ICD implanted in 157 patients $(57.7 \%$ of the cohort). During the $4.8 \pm 2.3$-year follow-up, the primary outcome occurred in 49 patients $(18.5 \%)$. The study demonstrated that $\mathrm{EF}<35 \%$ and sustained ventricular tachycardia induction during the electrophysiological study were independent predictors for primary outcome. Among patients with $\mathrm{EF} \geq 35 \%$ who induced sustained ventricular tachycardia during the electrophysiological study the test showed a sensitivity of $72.7 \%$, a specificity of 63\%, a PPV of $24.2 \%$, an NPV of $93.5 \%$ and an area under the receiver operating characteristic (ROC) curve of 0.681. This study, together with the studies conducted by Gatzoulis et al. and Hilfiker et al., corroborates the low incidence of major arrhythmic outcome (sustained ventricular arrhythmia) in patients with electrophysiological study with negative $\mathrm{PVS}^{27,28}$.

\section{CONCLUSION}

The occurrence of SCD in patients with HFpEF may represent up to a quarter of the causes of death among these patients, and should be an important therapeutic target to be considered when treating these patients. The clinical therapy for $\mathrm{HFpEF}$ to date is poor, with respect to clinical trials with statistically significant power to reduce hard outcomes. Some risk score models have been shown to have a good discriminative power to identifying high risk patients for SCD, selecting those who could benefit from a more invasive investigation strategy, with electrophysiological study and VPS and subsequently, ICD implantation. At the present, there is still a lack of clinical data that could support this strategy in general, but efforts should be made to look for markers and clinical characteristics that could more clearly assist in identifying patients at high risk for SCD and possibly guide future therapy, with measures to prevent SCD and reduce the causes of death in this population. 


\section{REFERENCES}

1. Ponikowski P, Voors AA, Anker SD, Bueno H, Cleland JGF, Coats AJS, et al. 2016 ESC Guidelines for the diagnosis and treatment of acute and chronic heart failure: The Task Force for the diagnosis and treatment of acute and chronic heart failure of the European Society of Cardiology (ESC): Developed with the special contribution of the Heart Failure Association (HFA) of the ESC. Eur Heart J. 2016;37(27):2129-200. https://doi.org/10.1093/eurheartj/ehw128

2. Curtis LH, Whellan DJ, Hammill BG, Hernandez AF, Anstrom KJ, Shea AM, et al. Incidence and prevalence of heart failure in elderly persons, 1994-2003. Arch Intern Med. 2008;168(4):418-24. https://doi.org/10.1001/archinternmed.2007.80

3. Abraham WT, Fonarow GC, Albert NM, Stough WG, Gheorghiade M, Greenberg BH, et al. Predictors of in-hospital mortality in patients hospitalized for heart failure: Insights from the Organized Program to Initiate Lifesaving Treatment in Hospitalized Patients with Heart Failure (OPTIMIZE-HF). J Am Coll Cardiol. 2008;52(5):347-56. https://doi.org/10.1016/j.jacc.2008.04.028

4. Loehr LR, Rosamond WD, Chang PP, Folsom AR, Chambless LE. Heart Failure incidence and survival (from The Atherosclerosis Risk in Communities Study). Am J Cardiol. 2008;101(7):1016-22. https://doi.org/10.1016/j.amjcard.2007.11.061

5. Vaduganathan M, Michel A, Hall K, Mulligan C, Nodari S, Shah SJ, et al. Spectrum of epidemiological and clinical findings in patients with heart failure with preserved ejection fraction stratified by study design: a systematic review. Eur J Heart Fail. 2016;18(1):54-65. https://doi.org/10.1002/ejhf.442

6. Owan TE, Hodge DO, Herges RM, Jacobsen SJ, Roger VL, Redfield MM. Trends in prevalence and outcome of heart failure with preserved ejection fraction. N EngI J Med. 2006;355(3):251-9. https://doi.org/10.1056/NEJMoa052256

7. Bursi F, Weston SA, Redfield MM, Jacobsen SJ, Pakhomov S, Nkomo VT, et al. Systolic and diastolic heart failure in the community. JAMA. 2006;296(18):2209-16. https://doi.org/10.1001/jama.296.18.2209

8. Meta-analysis Global Group in Chronic Heart Failure (MAGGIC). The survival of patients with heart failure with preserved or reduced left ventricular ejection fraction: an individual patient data meta-analysis. Eur Heart J. 2012;33(14):1750-7. https://doi.org/10.1093/ eurheartj/ehr254

9. Pitt B, Pfeffer MA, Assmann SF, Boineau R, Anand IS, Claggett B, et al. Spironolactone for heart failure with preserved ejection fraction. N Engl J Med. 2014;370(15):1383-92. https://doi.org/10.1056/NEJMoa1313731

10. Solomon SC, Wang D, Finn P, Skali H, Zornoff L, McMurray JJV, et al. Effect of candesartan on cause-specific mortality in heart failure patients: The Candesartan in Heart Failure Assessment of Reduction in Mortality and Morbidity (CHARM) program. Circulation. 2004;110(15):2180-3. https://doi.org/10.1161/01.CIR.0000144474.65922.AA

11. Zile MR, Gaasch WH, Anand IS, Haass M, Little WC, Miller AB, et al. Mode of death in patients with heart failure and a preserved ejection fraction: Results from the Irbesartan in Heart Failure with Preserved Ejection Fraction Study (I-Preserve) Trial. Circulation. 2010;121(12):1393-405. https://doi.org/10.1161/CIRCULATIONAHA.109.909614

12. Adabag S, Smith LG, Anand IS, Berger AK, Luepker RV. Sudden cardiac death in heart failure patients with preserved ejection fraction. J Card Fail. 2012;18(10):749-54. https://doi.org/10.1016/j.cardfail.2012.08.357

13. Hamaguchi S, Kinugawa S, Sobirin MA, Goto D, Tsuchihashi-Makaya M, Yamada S, et al. Mode of Death in Patients with Heart Failure and Reduced vs. Preserved Ejection Fraction: Report from the Registry of Hospitalized Heart Failure Patients. Circ J. 2012;76(7):16629. https://doi.org/10.1253/circj.CJ-11-1355

14. Vaduganathan M, Claggett BL, Chatterjee NA, Anand IS, Sweitzer NK, Fang JC, et al. Sudden death in heart failure with preserved ejection fraction: a competing risks analysis from the TOPCAT Trial. JACC Heart Fail. 2018;6(8):653-61. https://doi.org/10.1016/j. jchf.2018.02.014 2018

15. Adabag S, Rector TS, Anand IS, McMurray JJV, Zile M, Komajda M, et al. A prediction model for sudden cardiac death in patients with heart failure and preserved ejection fraction. Eur J Heart Fail. 2014;16(11):1175-82. https://doi.org/10.1002/ejhf.172

16. Packer DL, Prutkin JM, Hellkamp AS, Mitchell LB, Bernstein RC, Wood F, et al. Impact of implantable cardioverter-defibrillator, amiodarone, and placebo on the mode of death in stable patients with heart failure: analysis from the sudden cardiac death in heart failure trial. Circulation. 2009;120(22):2170-6. https://doi.org/10.1161/CIRCULATIONAHA.109.853689

17. Bardy GH, Lee KL, Mark DB, Poole JE, Packer DL, Boineau R, et al. Amiodarone or an Implantable Cardioverter-Defibrillator for Congestive Heart Failure. N Engl J Med. 2005;352(3):225-37. https://doi.org/10.1056/NEJMoa043399

18. Adabag S, Patton KK, Buxton AE, Rector TS, Ensrud KE, Vakil K, et al. Association of implantable cardioverter defibrillators with survival in patients with and without improved ejection fraction: Secondary Analysis of the Sudden Cardiac Death in Heart Failure Trial. JAMA Cardiol. 2017;2(7):767-74. https://doi.org/10.1001/jamacardio.2017.1413 
19. Adabag S, Langsetmo L. Sudden cardiac death risk prediction in heart failure with preserved ejection fraction. Heart Rhythm. 2020;17(3):358-64. https://doi.org/10.1016/j.hrthm.2019.12.009

20. Stecker EC, Vickers C, Waltz J, Socoteanu C, John BT, Mariani R, et al. Population-based analysis of sudden cardiac death with and without left ventricular systolic dysfunction: two-year findings from the Oregon Sudden Unexpected Death Study. J Am Coll Cardiol. 2006;47(6):1161-6. https://doi.org/10.1016/j.jacc.2005.11.045

21. Singh SN, Fisher SG, Carson PE, Fletcher RD, Department of Veterans Affairs CHF STAT Investigators. Prevalence and significance of nonsustained ventricular tachycardia in patients with premature ventricular contractions and heart failure treated with vasodilator therapy. J Am Coll Cardiol. 1998;32(4):942-7. https://doi.org/10.1016/S0735-1097(98)00338-6

22. Teerlink JR, Jalaluddin M, Anderson S, Kukin ML, Eichhorn EJ, Francis G, et al. Ambulatory ventricular arrhythmias in patients with heart failure do not specifically predict an increased risk of sudden death. Circulation. 2000;101(1):40-6. https://doi.org/10.1161/01. CIR.101.1.40

23. Doval HC, Nul DR, Grancelli HO, Varini SD, Soifer S, Corrado G, et al. Nonsustained ventricular tachycardia in severe heart failure: Independent marker of increased mortality due to sudden death. Circulation. 1996;94(12):3198-203. https://doi.org/10.1161/01. CIR.94.12.3198

24. Clementy N, Bisson A, Challal F, Andre C, Pierre B, Fauchier L, et al. Nonsustained ventricular tachycardia at the time of implantation predicts appropriate therapies on rapid ventricular arrhythmia in primary prevention patients with nonischemic cardiomyopathy: Results from the very-high-rate registry. JACC Clin Electrophysiol. 2017;3(11):1338-9. https://doi.org/10.1016/j.jacep.2017.04.016

25. Zhou Y, Zhao S, Chen K, Hua W, Su Y, Chen S, et al. Predictive value of rapid-rate non-sustained ventricular tachycardia in the occurrence of appropriate implantable cardioverter-defibrillator therapy. J Interv Card Electrophysiol. 2020;57(3):473-80. htttps:// doi.org/10.1007/s10840-019-00557-4

26. Gutierrez A, Ash J, Alexy T, Akdemir B, Cogswell R, Adabag S. Abstract 11898: Heart failure with preserved ejection fraction is a highly arrhythmogenic disease. Circulation. 2019;140(1 Suppl):A11898.

27. Gatzoulis KA, Tsiachris D, Arsenos P, Antoniou C-K, Dilaveris P, Sideris S, et al. Arrhythmic risk stratification in post-myocardial infarction patients with preserved ejection fraction: The PRESERVE EF Study. Eur Heart J. 2019;40(35):2940-9. https://doi. org/10.1093/eurheartj/ehz260

28. Hilfiker G, Schoenenberger AW, Erne P, Kobza R. Utility of electrophysiological studies to predict arrhythmic events. World J Cardiol. 2015;7(6):344-50. https://doi.org/10.4330/wjc.v7.i6.344 